\title{
Survivin expression in lung cancer: Association with smoking, histological types and pathological stages
}

\author{
HIROSHI HIRANO ${ }^{1}$, HAJIME MAEDA ${ }^{2}$, TOSHIHIKO YAMAGUCHI ${ }^{3}$, \\ SOICHIRO YOKOTA ${ }^{3}$, MASAHIDE MORI ${ }^{3}$ and SABURO SAKODA ${ }^{3}$
}

Departments of ${ }^{1}$ Pathology, ${ }^{2}$ Surgery and ${ }^{3}$ Internal Medicine, Toneyama National Hospital, Toyonaka, Osaka 560-8552, Japan

Received July 14, 2014; Accepted April 14, 2015

DOI: $10.3892 / \mathrm{ol} .2015 .3374$

\begin{abstract}
Survivin is expressed in the nucleus and/or cytoplasm of various malignant cells. Nuclear survivin is critical for the completion of mitosis, while cytoplasmic survivin functions as an inhibitor of apoptosis. The expression of survivin has been reported to be associated with the aggressiveness of certain types of cancer. The present study examined the association between cigarette smoking history and the expression of survivin and Ki-67 in lung adenocarcinomas of pathological (p) stages I, II and III. The expression of survivin and Ki-67 in adenocarcinomas was also compared with that of other p-stage I lung cancers, including squamous cell carcinoma (SqCC), large cell neuroendocrine carcinoma (LCNEC) and small cell carcinoma (SmCCs), of patients with a smoking history. In adenocarcinomas at p-stage I, labeling indices (LIs) of nuclear survivin and Ki-67 were significantly higher in tissue samples from smokers than those from non-smokers; however, the nuclear survivin and $\mathrm{Ki}-67 \mathrm{LIs}$ in p-stage II and III adenocarcinomas from non-smokers and smokers were similar to those in p-stage I adenocarcinomas of smokers. The nuclear survivin and $\mathrm{Ki}-67 \mathrm{LIs}$ in adenocarcinomas of smokers at p-stage I were lower than those in SqCCs, LCNECs and SmCCs of smokers at the same stage. Smokers with adenocarcinoma also exhibited a higher survival rate compared with that of smokers with SqCCs, LCNECs and SmCCs. The present results indicated that a history of smoking is associated with increased nuclear survivin and $\mathrm{Ki}-67$ expression in lung adenocarcinomas of p-stage I, but not p-stages II or III. In
\end{abstract}

Correspondence to: Dr Hiroshi Hirano, Department of Pathology, Toneyama National Hospital, 1-1, Toneyama 5 chome, Toyonaka, Osaka 560-8552, Japan

E-mail: hi-hi-hirano@hotmail.com

Abbreviations: IAP, inhibitor of apoptosis protein; BIR, baculovirus IAP repeat; NES, nuclear export signal; NLS, nuclear localization signal; $\mathrm{Crm1}$, chromosome region maintenance protein 1; INCENP, inner centromere protein; SqCC, squamous cell carcinoma; LCNEC, large cell neuroendocrine carcinoma; SmCC, small cell carcinoma.

Key words: survivin, lung cancer, smoking, histological type, Ki-67 addition it was revealed that, in smokers, the nuclear survivin and $\mathrm{Ki}$-67 expression in p-stage I adenocarcinomas was lower than that of other p-stage I lung cancer types, and was associated with an enhanced survival rate. In conclusion, smoking is associated with the histogenesis of lung adenocarcinoma but not with the development of lung adenocarcinoma, based on the nuclear expression levels of Ki-67 and survivin.

\section{Introduction}

Survivin was first identified in 1997 as a member of the inhibitor of apoptosis protein (IAP) family, which contain a family-specific baculovirus IAP repeat (BIR) (1). This BIR domain exists at the N-terminal and is associated with the inhibition of apoptosis (2). The human survivin gene is located on chromosome 17 (17q25) (3). Survivin is expressed in the nucleus and/or cytoplasm of various malignant tumor cells (4), and is also expressed in fetal and certain proliferating adult tissues, although remains undetectable in differentiated tissues (5). In the cytoplasm, survivin functions as an inhibitor of apoptosis, while in the nucleus, survivin regulates cell proliferation (6).

Survivin possesses a nuclear export signal (NES), which allows it to bind to its export receptor, chromosome region maintenance protein 1 (Crm1). In the nucleus, survivin forms a complex with aurora kinase $\mathrm{B}$, the inner centromere protein (INCENP) and borealin to complete mitosis. Crm1 is critical in tethering this complex to the centromere (Fig. 1) (7). At the end of mitosis, Crm1 is released from this complex and exports survivin from the nucleus to the cytoplasm, where it functions as an inhibitor of apoptosis.

Human survivin has multiple splice variants, which have various functions and localizations; wild type survivin (WT-survivin), survivin-2a, survivin-2B, survivin- $\Delta \mathrm{Ex} 3$ and survivin-3B have been identified to date (Fig. 2) (8). Survivin- $\Delta \mathrm{Ex} 3$ and survivin-2a lack a nuclear localization signal (NLS), and remain in the cytoplasm, while WT-survivin, survivin-2B and survivin-3B possess an NLS and are able to function in the completion of mitosis in the nucleus (9).

Various types of cancer express survivin. As survivin has a dual role as an apoptosis inhibitor and a mitotic effector, the expression of survivin in cancer cells may protect them from therapeutic drug-induced apoptosis and promote their proliferation. The association between the aggressiveness of 
cancer cells and the expression of survivin has been investigated in numerous types of cancer (10-12). However, various studies have reported inconsistent findings with regard to the significance of survivin as a prognostic factor for cancer patients. Nuclear survivin expression has been reported to be correlated with poor prognosis in patients with various types of cancer, including hepatocellular carcinoma, esophageal squamous cell carcinoma, as well as urinary bladder and ovarian cancer (12-15). However, it has been reported to be a valuable prognostic factor in other types of cancer, for example gastric cancer and invasive breast cancer $(10,16)$. With regard to lung cancer, nuclear survivin expression has been reported to be a useful prognostic factor for patients with advanced non-small cell cancers (stages III and IV), and a poor prognostic factor for patients with non-small cell carcinomas at earlier stages (I and II). Although a number of studies have investigated survivin expression in non-small cell lung carcinomas $(11,17,18)$, little data is available with regard to survivin expression in lung cancers of various histological types at various stages, or the association between survivin expression and smoking history. The paraffin-embedded blocks of lung tumor tissue removed during surgeries performed in Toneyama National Hospital (Toyonaka, Osaka, Japan) between 2002 and 2011 included sufficient numbers to allow statistical analysis of adenocarcinomas from smokers and non-smokers at p-stages I, II and III, as well as squamous cell carcinomas (SqCCs), large cell neuroendocrine carcinomas (LCNECs) and small cell carcinomas (SmCCs) from smokers at $\mathrm{p}$-stage I. Among the proliferative markers of tumors, the Ki-67 labeling index (Ki67-LI) is widely accepted as one of the most reliable markers for estimating the malignancy grade and the prognosis of various types of tumor (19). The Ki-67 LI of non-small cell lung carcinoma has prognostic value (20). The present study investigated the expression of survivin and $\mathrm{Ki}-67$ in these tumors.

\section{Materials and methods}

Patients and specimens. Paraffin-embedded tissue blocks of adenocarcinomas of p-stages I, II and III, as well as SqCCs, LCNECs and SmCCs at p-stage I, were selected. All tissues were obtained during surgeries performed at Toneyama National Hospital (Osaka, Japan) from January 2002 to December 2011. None of the patients whose tissues were used in the present study had received chemotherapy or radiotherapy prior to surgery. The numbers of samples obtained are presented in Table I. The Brinkman index (BI), which is described in Table I represents the number of pieces of tobacco smoked/day, multiplied by the number of years smoking history. The mortality of lung cancer patients with a $\mathrm{BI} \geq 400$ is $\sim 4.9$ times higher compared with that of non-smoking patients (21). In the present study, the majority of the patients with SqCC, LCNEC and SmCC had a high BI, and the poor prognosis of those histological types is possibly due to an increased BI. The adenocarcinoma samples were almost evenly distributed between p-stages I, II and III, and were identified in non-smokers and smokers (including current smokers and ex-smokers) (Table I). By contrast, the majority of SqCCs, LCNECs and SmCCs were of p-stage I and almost all patients who had

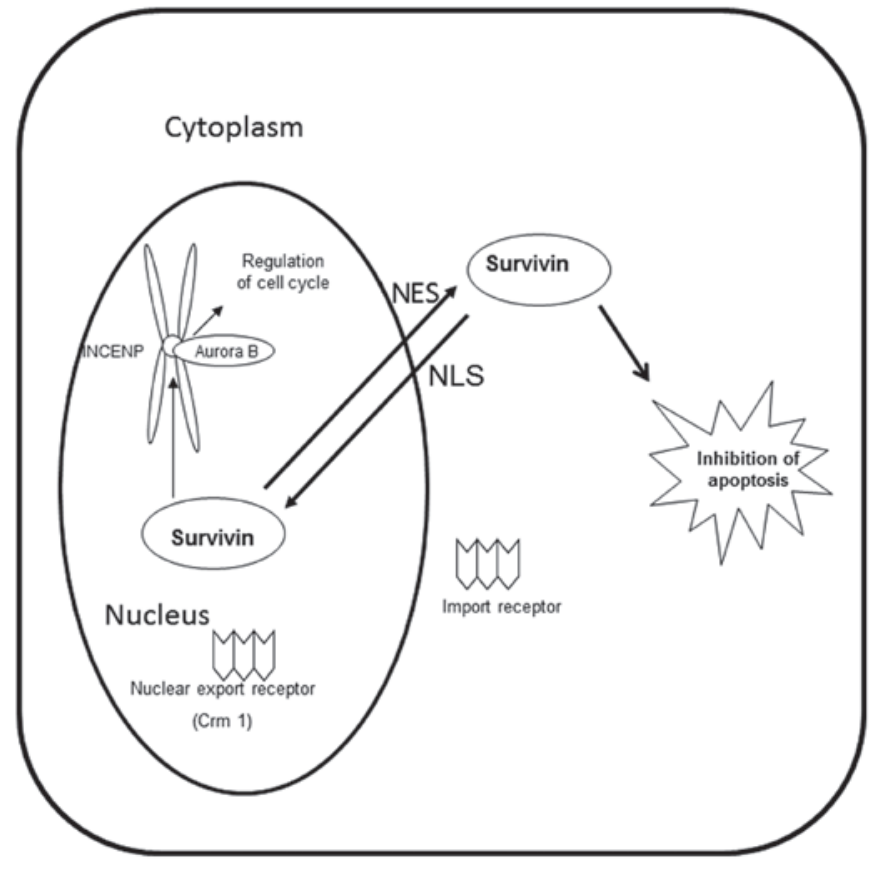

Figure 1. Functions, localization and transport of survivin. INCENP, inner centromere protein; NES, nuclear export signal; NLS, nuclear localization signal; Crm1, chromosome region maintenance protein 1.

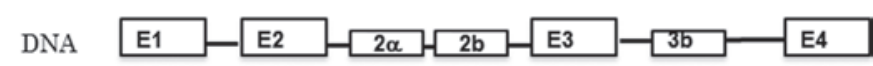

Survivin variant

WT-Survivin

\begin{tabular}{|l|l|l|l|}
\hline E1 & E2 & E3 & E4 \\
\hline
\end{tabular}

Survivin- $\Delta E \times 3$

\begin{tabular}{|l|l|l|}
\hline E1 & E2 & E4 \\
\hline
\end{tabular}

Survivin- $2 \alpha$

\begin{tabular}{|l|l|l|}
\hline E1 & E2 & $2 \alpha$ \\
\hline
\end{tabular}

Survivin-2B

\begin{tabular}{|l|l|l|l|l|}
\hline E1 & E2 & 2b & E3 & E4 \\
\hline
\end{tabular}

Survivin-3B

\begin{tabular}{|l|l|l|l|}
\hline E1 & E2 & 3b & E3 \\
\hline
\end{tabular}

Figure 2. Human survivin splice variants. E, exon; WT, wild type.

undergone surgery for these types of tumor were smokers (Table I).

The paraffin-embedded tissue blocks used for this study were produced following the fixation of tumor tissues in $0.01 \mathrm{M}$ phosphate-buffered $10 \%$ formalin (pH 7.4). Clinical data, including follow-up findings, were available for all cases. Written informed consent from each patient, allowing the arbitrary use of tumor tissues for pathological studies, was obtained prior to surgery. The present study was approved by the ethics committee of Toneyama National Hospital (approval number 1334).

Immunohistochemistry. For immunohistochemical examinations of survivin, one representative tissue block from each tumor was used, with $5-\mathrm{mm}$ sections prepared. Immunohistochemical staining was performed using an avidin-streptavidin immunoperoxidase method with a rabbit polyclonal anti-human survivin antibody (Novus Biologicals, 
Table I. Clinical features of patients with various subtypes of lung cancer.

A, Adenocarcinoma $(\mathrm{n}=87)$

\begin{tabular}{|c|c|c|c|}
\hline Characteristic & Stage I & Stage II & Stage III \\
\hline Patients, $\mathrm{n}$ & 34 & 22 & 31 \\
\hline \multicolumn{4}{|l|}{ Age, years } \\
\hline Mean \pm SE & $66.4 \pm 1.5$ & $66.6 \pm 1.8$ & $65.8 \pm 1.2$ \\
\hline Range & $50-80$ & $53-80$ & $47-81$ \\
\hline \multicolumn{4}{|l|}{ Gender, $\mathrm{n}$} \\
\hline Male & 20 & 15 & 18 \\
\hline Female & 14 & 7 & 13 \\
\hline \multicolumn{4}{|l|}{ Smoking status, $\mathrm{n}$} \\
\hline Non-smoker & 13 & 4 & 8 \\
\hline Smoker & 21 & 18 & 23 \\
\hline $\mathrm{BI}$ of smoker, mean $\pm \mathrm{SE}$ & $921.2 \pm 57.5$ & $829.2 \pm 108.0$ & $1137.6 \pm 137.9$ \\
\hline
\end{tabular}

B, Squamous cell carcinoma $(n=44)$

\begin{tabular}{|c|c|c|c|}
\hline Characteristic & Stage I & Stage II & Stage III \\
\hline Patients, $\mathrm{n}$ & 33 & 2 & 9 \\
\hline \multicolumn{4}{|l|}{ Age, years } \\
\hline Mean \pm SE & $68.4 \pm 1.5$ & $66.5 \pm 7.6$ & $70.9 \pm 3.4$ \\
\hline Range & $55-79$ & $59-74$ & $48-82$ \\
\hline \multicolumn{4}{|l|}{ Gender, $\mathrm{n}$} \\
\hline Male & 30 & 2 & 7 \\
\hline Female & 3 & 1 & 2 \\
\hline \multicolumn{4}{|l|}{ Smoking status, $\mathrm{n}$} \\
\hline Non-smoker & 1 & 0 & 1 \\
\hline Smoker & 32 & 2 & 8 \\
\hline $\mathrm{BI}$ of smoker, mean $\pm \mathrm{SE}$ & $1179.4 \pm 83.7$ & & $935.6 \pm 231.1$ \\
\hline
\end{tabular}

C, Large cell neuroendocrine carcinoma $(n=13)$

\begin{tabular}{lcc}
\hline Characteristic & Stage I & Stage II \\
\hline Patients, $\mathrm{n}$ & 13 & 0 \\
Age, years & & \\
Mean \pm SE & $69.3 \pm 2.8$ & \\
Range & $58-79$ & \\
Gender, $\mathrm{n}$ & 12 & \\
Male & 1 & \\
Female & 1 & \\
Smoking status, $\mathrm{n}$ & 12 & \\
Non-smoker & $1012.9 \pm 118.0$ \\
Smoker & \\
BI of smoker, mean \pm SE &
\end{tabular}

D, Small cell carcinoma $(n=13)$

\begin{tabular}{lccc}
\hline Characteristic & Stage I & Stage II & Stage III \\
\hline Patients, $\mathrm{n}$ & 10 & 2 & 1
\end{tabular}


Table I. Continued.

\begin{tabular}{|c|c|c|c|}
\hline Characteristic & Stage I & Stage II & Stage III \\
\hline \multicolumn{4}{|l|}{ Age, years } \\
\hline Mean $\pm \mathrm{SE}$ & $69.1 \pm 2.9$ & & \\
\hline Range & $56-79$ & $62-73$ & 80 \\
\hline \multicolumn{4}{|l|}{ Gender, $\mathrm{n}$} \\
\hline Male & 9 & 2 & 0 \\
\hline Female & 1 & 1 & 1 \\
\hline \multicolumn{4}{|l|}{ Smoking status, $\mathrm{n}$} \\
\hline Non-smoker & 0 & 0 & 0 \\
\hline Smoker & 10 & 2 & 1 \\
\hline $\mathrm{BI}$ of smoker, mean $\pm \mathrm{SEM}$ & $948 \pm 127.4$ & & \\
\hline
\end{tabular}

SE, standard error of the mean; BI, Brinkman index.

Table II. Survivin expression and Ki-67 labeling indices in adenocarcinomas of non-smokers and smokers at p-stage I, II or III.

\begin{tabular}{|c|c|c|c|}
\hline Stage & Non-smokers & Smokers & Difference \\
\hline Stage I, n & 13 & 21 & \\
\hline Survivin nuclear labeling index, $\%$ & $2.9 \pm 1.0$ & $9.7 \pm 2.4$ & $\mathrm{P}<0.05$ \\
\hline Survivin cytoplasm expression $>10 \%, \mathrm{n}(\%)$ & $12(92.3)$ & $16(76.2)$ & NS \\
\hline Ki-67 labeling index, \% & $12.0 \pm 1.6$ & $20.5 \pm 2.8$ & $\mathrm{P}<0.05$ \\
\hline Stage II, $\mathrm{n}$ & 4 & 18 & \\
\hline Survivin nuclear labeling index, $\%$ & $9.0 \pm 2.7$ & $12.3 \pm 2.1$ & NS \\
\hline Survivin cytoplasm expression $>10 \%, \mathrm{n}(\%)$ & $3(75.0)$ & $9(50.0)$ & NS \\
\hline Ki-67 labeling index, $\%$ & $17.8 \pm 4.5$ & $28.1 \pm 3.3$ & NS \\
\hline Stage III, n & 8 & 23 & \\
\hline Survivin nuclear labeling index, $\%$ & $7.1 \pm 1.8$ & $13.2 \pm 2.8$ & NS \\
\hline Survivin cytoplasm expression $>10 \%, \mathrm{n}(\%)$ & $7(87.5)$ & $12(52.2)$ & NS \\
\hline Ki-67 labeling index, $\%$ & $15.1 \pm 2.6$ & $21.9 \pm 2.1$ & NS \\
\hline
\end{tabular}

Nuclear survivin and Ki-67 labeling indices are presented as the mean \pm standard error of the mean. NS, not significant.

Littleton, CO, USA) at a 1:500 dilution, or with a prediluted anti-human Ki-67 mouse monoclonal antibody (Dako, Glostrup, Denmark). The primary antibodies were detected using the iView DAB universal kit (Ventana Medical Systems Inc., Tucson, AZ, USA), which is a detection kit including from a mixture of anti-mouse Ig and anti-rabbit Ig biotinylated secondary antibodies using DAB/H2O2 as substrates. Antigen retrieval was conducted by incubation of deparaffinized sections in cell condition 1 solution from the aforementioned kit for $64 \mathrm{~min}$ at $100^{\circ} \mathrm{C}$ and immunohistochemical staining was conducted using an automated Benchmark system (Ventana Medical System, Tuscon, AZ, USA), according to the manufacturer's instructions. To estimate the labeling index (LI) of nuclear survivin or Ki-67 in each tumor, 1,000 nuclei stained positively or negatively were counted automatically, using Win Roof software (Mitani Co, Tokyo, Japan). To determine positive staining of cytoplasmic survivin, tumors were classified into two groups based on the percentage of positively stained cells: $>10 \%$, positive staining; and $<10 \%$, negative staining.

Statistical analysis. Statistical analyses were performed using the Excel Statistics 2012 software package for Windows (SSRI, Tokyo, Japan). $\mathrm{P}<0.05$ was considered to indicate a statistically significant difference. Categorical data was analyzed using a $\chi^{2}$ test. Data comprising multiple values are presented as the mean \pm standard error of the mean and these data were analyzed using Bonferroni's multiple comparison test. The survival curves were analyzed by the Kaplan-Meier method, followed by the Log rank test.

\section{Results}

Survivin expression in adenocarcinomas of non-smokers and smokers. In adenocarcinomas at p-stage I, the nuclear survivin LIs were significantly higher for smokers than for non-smokers 
Table III. Survivin expression and Ki-67 labeling indices in various histological types of lung cancer in smokers.

\begin{tabular}{lcccr}
\hline Stage & Adenocarcinoma & SqCC & LCNEC & SmCC \\
\hline Stage I, n & 21 & 32 & 12 & 10 \\
Survivin nuclear labeling index, \% & $9.7 \pm 2.4^{\mathrm{b}, \mathrm{c}, \mathrm{d}}$ & $29.1 \pm 2.9^{\mathrm{a}, \mathrm{c}}$ & $41.5 \pm 2.3^{\mathrm{a}, \mathrm{b}}$ & $33.3 \pm 3.2^{\mathrm{a}}$ \\
Survivin cytoplasm expression $>10 \%, \mathrm{n}(\%)$ & $16(76.2)^{\mathrm{c}, \mathrm{d}}$ & $29(90.6)^{\mathrm{c}, \mathrm{d}}$ & $3(25.0)^{\mathrm{a}, \mathrm{b}}$ & $3(30.0)^{\mathrm{a}, \mathrm{b}}$ \\
Ki-67 labeling index, $\%$ & $20.5 \pm 2.8^{\mathrm{b}, \mathrm{d}, \mathrm{d}}$ & $45.5 \pm 2.7^{\mathrm{a}}$ & $46.9 \pm 7.4^{\mathrm{a}}$ & $54.2 \pm 4.0^{\mathrm{a}}$ \\
Stage III, n & 23 & 8 & & \\
Survivin nuclear labeling index, \% & $13.2 \pm 2.8$ & $19.4 \pm 4.2$ & & \\
Survivin cytoplasm expression $>10 \%, \mathrm{n}(\%)$ & $12(52.2)$ & $7(87.5)$ & & \\
Ki-67 labeling index, $\%$ & $21.9 \pm 2.1^{\mathrm{b}}$ & $32.2 \pm 4.5^{\mathrm{a}}$ & & \\
\end{tabular}

SqCC, squamous cell carcinoma; LCNEC, large cell neuroendocrine carcinoma; SmCC, small cell carcinoma. Nuclear survivin and Ki-67 labeling indices are presented as the mean \pm standard error of the mean. ${ }^{a} \mathrm{P}<0.05$ vs. adenocarcinoma; ${ }^{b} \mathrm{P}<0.05 \mathrm{vs}$. $\mathrm{SqCC}$; ${ }^{\mathrm{C}}<0.05 \mathrm{vs}$. LCNEC; ${ }^{\mathrm{d}} \mathrm{P}<0.05$ vs. SmCC.
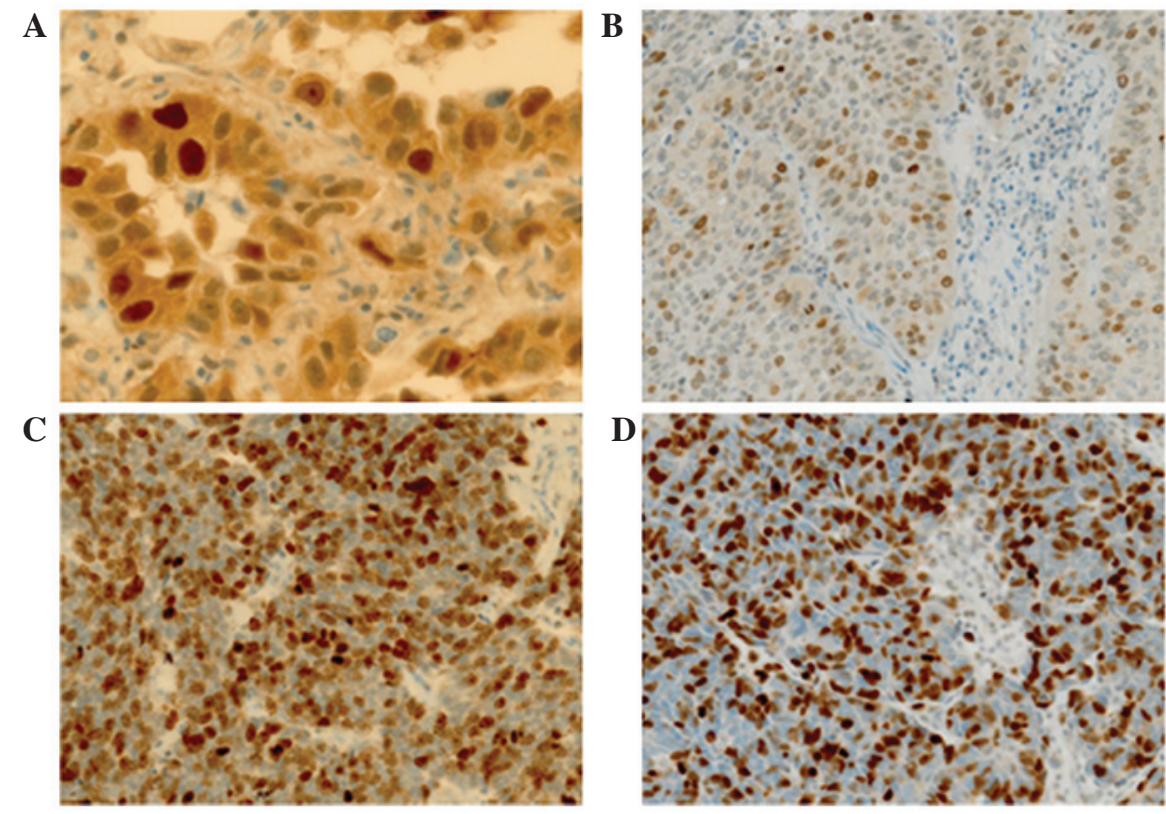

Figure 3. Immunohistochemical staining of survivin in p-stage I lung carcinomas: (A) Nuclear and cytoplasmic expression in adenocarcinoma (x40 magnification); (B) nuclear expression in squamous cell carcinoma (x20 magnification); (C) nuclear expression in small cell carcinoma (x20 magnification); (D) nuclear expression in large cell neuroendocrine carcinoma (x20 magnification).

$(\mathrm{P}<0.05)$, while there was no significant difference in the cytoplasmic survivin expression between adenocarcinomas from smokers and non-smokers (Table II; Fig. 3A). The Ki-67 LIs of the adenocarcinomas of smokers were also significantly higher compared with those of the adenocarcinomas of non-smokers. In adenocarcinomas at p-stage II and III, smoking history was not significantly associated with the nuclear survivin LI, cytoplasmic survivin expression or Ki-67 LI. The nuclear survivin LIs and Ki-67 LIs in adenocarcinomas at p-stage II and III were similar to those in adenocarcinomas of smokers at p-stage I.

Survivin expression in lung cancers of various histological types. Survivin expression and Ki-67 LIs were compared between adenocarcinomas and other histological types of lung carcinoma, including SqCC, LCNEC and SmCC, from smokers at p-stage I, as almost all patients who had undergone

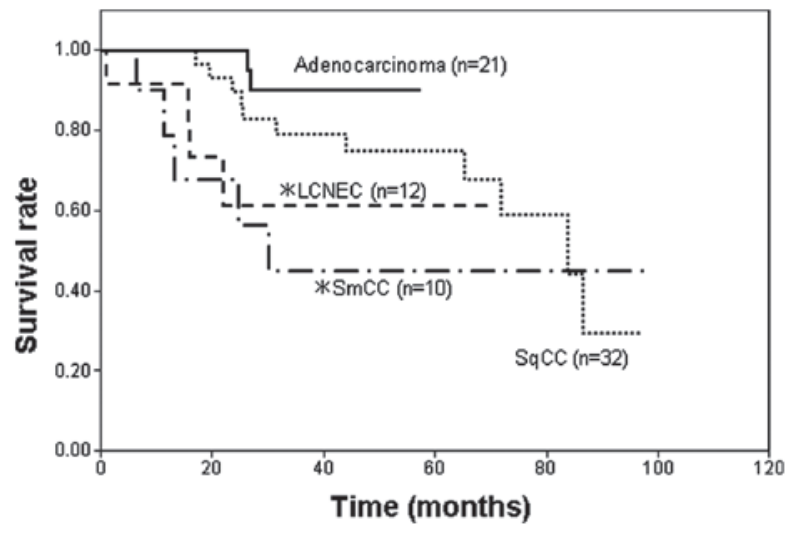

Figure 4. Survival curves of patients with a history of smoking with various histological types of lung carcinoma at p-stage I. LCNEC, large cell neuroendocrine carcinoma; SmCC, small cell carcinoma; $\mathrm{SqCC}$, squamous cell carcinoma. ${ }^{*} \mathrm{P}<0.05$ vs. adenocarcinoma. 
surgery for the treatment of SqCC, LCNEC and SmCC were smokers (Table II). The nuclear survivin and Ki-67 LIs in the adenocarcinoma samples were significantly lower compared with those of the SqCC, LCNEC and SmCC samples $(\mathrm{P}<0.05$; Table III; Fig. 3B, C and D). By contrast, the cytoplasmic survivin expression in SqCC samples was similar to that of adenocarcinoma samples, whilst it was decreased in LCNEC and SmCC compared with adenocarcinoma.

Survival rate of patients with different histological types of lung carcinoma. When the survival rates were compared among smokers with adenocarcinoma, SqCC, LCNEC and SmCC at p-stage I, the survival rates of smokers with LCNEC and SmCC were significantly poorer compared with that of smokers with adenocarcinoma (Fig. 4). The survival rate of SqCC patients was also poorer than that of adenocarcinoma patients, however, this difference was not statistically significant. The duration of the follow-up for the p-stage I adenocarcinoma patients was shorter compared with the other type of lung carcinoma patients, since p-stage I adenocarcinoma has a more favorable prognosis; therefore, a 5-year follow-up was considered to be sufficient.

In conclusion, the present study indicates that smoking is associated with the histogenesis of lung adenocarcinoma but not with the development of lung adenocarcinoma, based on the nuclear expression levels of Ki-67 and survivin in lung cancer tissue samples.

\section{Discussion}

In adenocarcinomas at p-stage I, the LIs of nuclear survivin and $\mathrm{Ki}-67$ were significantly higher in the adenocarcinomas of smokers than in adenocarcinomas of non-smokers. These results are consistent with the results of a previous study by our group, in which an association was identified between smoking and nuclear survivin expression and $\mathrm{Ki}-67 \mathrm{LI}$ in p-stage IA adenocarcinomas (18). Dasgupta et al (22) have reported that the cigarette component nicotine upregulates survivin expression in human non-small cell carcinoma cell lines. Therefore, it is conceivable that smoking induces survivin expression at an early developmental stage of lung adenocarcinoma. The increased nuclear survivin expression in the adenocarcinomas of smokers compared with that of non-smokers may be associated with the higher Ki-67 LIs in these adenocarcinomas. Maeda et al (23) reported that smoking was associated with poor outcomes in patients with clinical stage IA adenocarcinomas. The poor prognoses of these patients may be associated with the increased nuclear survivin expression.

Notably, nuclear survivin and Ki-67 LIs, in adenocarcinomas at p-stages II and III of non-smokers and smokers were similar to those in p-stage I adenocarcinomas of smokers. These results indicate that nuclear survivin expression and proliferative activity may be increased during the progression of disease following the early developmental stages of adenocarcinoma.

Nuclear survivin and Ki-67 LIs in adenocarcinomas of smokers at p-stage I were significantly lower compared with those of SqCCs, LCNECs and SmCCs of smokers at the same stage. These results suggested that survivin expression may depend on the histological type of lung cancer, at least at p-stage I. The higher Ki-67 LIs in SqCCs, LCNECs and SmCCs may be associated with higher nuclear survivin expression in these histological types of lung cancer. Inconsistent with the higher Ki-67 LIs in SqCCs, LCNECs and SmCCs compared with that of adenocarcinomas of smokers at p-stage I, the survival rates of smokers with SqCCs, LCNECs and SmCCs were poorer than those of smokers with adenocarcinoma.

In contrast to the nuclear expression of survivin, cytoplasmic survivin expression was not increased in non-adenocarcinoma lung cancer types compared with that in adenocarcinoma, and the poorer survival rates of patients with these types of cancer were not associated with its increase. Therefore, cytoplasmic survivin expression may have little effect on the proliferation or prognosis of lung cancer.

In conclusion, the present results suggest that a history of smoking is accompanied by an increase in nuclear survivin and $\mathrm{Ki}-67$ expression in lung adenocarcinomas at p-stage I, but not at p-stage II and III; and that nuclear survivin and $\mathrm{Ki}-67$ expression in adenocarcinomas of smokers at p-stage I is lower than that in other types of lung cancer of smokers at p-stage I, which is associated with an enhanced survival rate.

\section{Acknowledgements}

The authors would like to thank Mr. Akira Kimura and Mr. Hiroshi Yamada (Laboratory Medicine, Toneyama National Hospital) for their technical assistance, and Ms. Yuko Ito (Laboratory Medicine, Toneyama National Hospital) for providing secretarial assistance.

\section{References}

1. Ambrosini G, Adida C and Altieri DC: A novel anti-apoptosis gene, survivin, expressed in cancer and lymphoma. Nat Med 3: 917-921, 1997.

2. Srinivasula SM and Ashwell JD: IAPs: What's in a name? Mol Cell 30: 123-135, 2008.

3. Storlazzi CT, Brekke HR, Mandahl N, et al: Identification of a novel amplicon at distal 17q containing the BIRC5/SURVIVIN gene in malignant peripheral nerve sheath tumours. J Pathol 209: 492-500, 2006.

4. Connell CM, Colnaghi R and Wheatley SP: Nuclear survivin has reduced stability and is not cytoprotective. J Biol Chem 283: 3289-3296, 2008.

5. Salvesen GS and Duckett CS: IAP proteins: Blocking the road to death's door. Nat Rev Mol Cell Biol 3: 401-410, 2002.

6. Stauber RH, Mann W and Knauer SK: Nuclear and cytoplasmic survivin: Molecular mechanism, prognostic, and therapeutic potential. Cancer Res 67: 5999-6002, 2007.

7. Knauer SK, Mann W and Stauber RH: Survivin's dual role: An export's view. Cell Cycle 6: 518-521, 2007.

8. Mull AN, Klar A and Navara CS: Differential localization and high expression of SURVIVIN splice variants in human embryonic stem cells but not in differentiated cells implicate a role for SURVIVIN in pluripotency. Stem Cell Res (Amst) 12: 539-549, 2014.

9. Li F and Ling X: Survivin study: An update of 'What is the next wave'? J Cell Physiol 208: 476-486, 2006.

10. Hernandez JM, Farma JM, Coppola D, et al: Expression of the antiapoptotic protein survivin in colon cancer. Clin Colorectal Cancer 10: 188-193, 2011.

11. Xie YL, An L, Jiang H and Wang J: Nuclear survivin expression is associated with a poor prognosis in Caucasian non-small cell lung cancer patients. Clin Chim Acta 414: 41-43, 2012.

12. Vivas-Mejia PE, Rodriguez-Aguayo C, Han HD, et al: Silencing survivin splice variant $2 \mathrm{~B}$ leads to antitumor activity in taxane-resistant ovarian cancer. Clin Cancer Res 17: 37163726, 2011.

13. Morinaga S, Nakamura Y, Ishiwa N, et al: Expression of survivin mRNA associates with apoptosis, proliferation and histologically aggressive features in hepatocellular carcinoma. Oncol Rep 12: 1189-1194, 2004 
14. Zhu H, Wang Q, Hu C, et al: High expression of survivin predicts poor prognosis in esophageal squamous cell carcinoma following radiotherapy. Tumour Biol 32: 1147-1153, 2011.

15. Sun YW, Xuan Q, Shu QA, et al: Correlation of tumor relapse and elevated expression of survivin and vascular endothelial growth factor in superficial bladder transitional cell carcinoma. Genet Mol Res 12: 1045-1053, 2013.

16. Vallböhmer D, Drebber U, Schneider PM, et al: Survivin expression in gastric cancer: Association with histomorphological response to neoadjuvant therapy and prognosis. J Surg Oncol 99: 409-413, 2009.

17. Ichiki Y, Hanagiri T, Takenoyama M, et al: Tumor specific expression of survivin-2B in lung cancer as a novel target of immunotherapy. Lung Cancer 48: 281-289, 2005.

18. Hirano H, Maeda H, Takeuchi Y, et al: Association of cigarette smoking with the expression of nuclear survivin in pathological Stage IA lung adenocarcinomas. Med Mol Morphol 47: 196-200, 2014
19. Gerdes J, Schwab U, Lemke H and Stein H: Production of a mouse monoclonal antibody reactive with a nuclear antigen associated with cell proliferation. Int J Cancer 31: 13-20, 1983.

20. Nguyen VN, Mirejovský P, Mirejovský T, et al: Expression of cyclin D1, Ki-67 and PCNA in non-small cell lung cancer: Prognostic significance and comparison with p53 and bcl-2. Acta Histochem 102: 323-338, 2000.

21. Maeshima AM, Tochigi N, Tsuta K, et al: Histological evaluation of the effect of smoking on peripheral small adenocarcinomas of the lung. J Thorac Oncol 3: 698-703, 2008.

22. Dasgupta P, Kinkade R, Joshi B, et al: Nicotine inhibits apoptosis induced by chemotherapeutic drugs by up-regulating XIAP and survivin. Proc Natl Acad Sci USA 103: 6332-6337, 2006.

23. Maeda R, Yoshida J, Ishii G, et al: Influence of cigarette smoking on survival and tumor invasiveness in clinical stage IA lung adenocarcinoma. Ann Thorac Surg 93: 1626-1632, 2012. 